\title{
Design, data management, and population baseline characteristics of the PERFORM magnetic resonance imaging project
}

\author{
P. Maeder $\cdot$ L. Bracoud $\cdot$ H. Chabriat $\cdot$ \\ A. Gass $\cdot$ P. Michel $\cdot$ M. Hennerici
}

Received: 12 August 2010/Revised: 22 October 2010/Accepted: 15 November 2010/Published online: 3 December 2010

(C) The Author(s) 2010. This article is published with open access at Springerlink.com

\begin{abstract}
Quantitative information from magnetic resonance imaging (MRI) may substantiate clinical findings and provide additional insight into the mechanism of clinical interventions in therapeutic stroke trials. The PERFORM study is exploring the efficacy of terutroban versus aspirin for secondary prevention in patients with a history of ischemic stroke. We report on the design of an exploratory longitudinal MRI follow-up study that was performed in a subgroup of the PERFORM trial. An international multicentre longitudinal follow-up MRI study was designed for different MR systems employing safety and efficacy readouts: new T2 lesions, new DWI lesions, whole brain volume change, hippocampal volume change, changes in tissue microstructure as depicted by mean diffusivity and
\end{abstract}

On behalf of the PERFORM Study Investigators. Investigators are listed in Appendix.

PERFORM: Prevention of cerebrovascular and cardiovascular Events of ischemic origin with teRutroban in patients with a history oF ischemic strOke or tRansient ischeMic attack.

P. Maeder ( $\square)$

Centre Hospitalier Universitaire Vaudois, Service de Radiologie,

Rue du Bugnon 47, 1011 Lausanne, Switzerland

e-mail: philippe.maeder@chuv.ch

L. Bracoud

BioClinica, Lyon, France

e-mail: luc.bracoud@bioclinica.com

H. Chabriat

Hôpital Lariboisière, Service de Neurologie, Paris, France

e-mail: hugues.chabriat@1rb.ap-hop-paris.fr

A. Gass · M. Hennerici

Department of Neurology, University of Heidelberg,

Mannheim, Germany fractional anisotropy, vessel patency on MR angiography, and the presence of and development of new microbleeds. A total of 1,056 patients (men and women $\geq 55$ years) were included. The data analysis included 3D reformation, image registration of different contrasts, tissue segmentation, and automated lesion detection. This large international multicentre study demonstrates how new MRI readouts can be used to provide key information on the evolution of cerebral tissue lesions and within the macrovasculature after atherothrombotic stroke in a large sample of patients.

Keywords MRI - Lesion load · Brain atrophy ·

Microbleeds · PERFORM - Longitudinal brain disease progression

\section{Introduction}

During the last 20 years, novel magnetic resonance imaging (MRI) techniques have emerged that allow the non-invasive

M. Hennerici

e-mail: hennerici@neuro.ma.uni-heidelberg.de

A. Gass

Department of Neurology, University Hospital Basel,

Basel, Switzerland

e-mail: AGass@uhbs.ch

P. Michel

Centre Hospitalier Universitaire Vaudois, Service de Neurologie,

Rue du Bugnon 47, 1011 Lausanne, Switzerland

e-mail: patrik.michel@chuv.ch 
and rapid assessment of brain functioning and cerebral tissue lesions [1]. The MRI is now utilized as the primary imaging modality in major stroke centres to detect and measure cerebral tissue lesions as well as changes in the vascular structure [2]. Moreover, it becomes an accepted feature of clinical trials in a variety of neurological disorders, and particularly in stroke. New MRI markers of cerebrovascular diseases (microbleeds, diffusion) have been recently evaluated offering reliable parameters of assessment of clinical outcome [3, 4]. There are few longitudinal neuro-radiological data assessed in large stroke trials, which mostly are based on the clinical evaluation. In this context the PERFORM (Prevention of Cerebrovascular and Cardiovascular Events of Ischemic Origin with Terutroban in Patients with a History of Ischemic Stroke or Transient Ischemic Attack) MRI project was designed to depict by MRI structural and functional information on the evolution of cerebral atherothrombotic processes after a recent ischemic stroke. The aim of this major sub study is to provide a longitudinal assessment of multiple MRI parameters in a large subgroup of a post stroke population with a follow-up of 2 years. The rationale and design of the PERFORM study, which includes 19,119 patients randomly allocated either to terutroban, a specific thromboxane prostaglandin (TP) receptor antagonist [5], $30 \mathrm{mg} /$ day, or to aspirin $100 \mathrm{mg} /$ day, have been described elsewhere, together with the characteristics of the whole population at baseline $[6,7]$. Herein we report the design, framework and conduct for this complex international multi-centre longitudinal study. In particular, the MRI methodology chosen and the data that can be obtained in such a large MRI study are presented.

\section{Design}

\section{Subjects}

The patients included in the study were aged $\geq 55$ years, had a cerebral or retinal infarction in the last 3 months preceding inclusion or TIA in the last 8 days, and were stable at inclusion. They did not have any MRI contraindication such as known risk for exposure to high magnetic fields (e.g., pacemakers, implanted ferromagnetic devices, metallic implants, metal fragments, cochlear implants, brain and spinal cord stimulators), severe claustrophobia and difficulty lying in supine position. MRI quality check performed at M1 was validated by the imaging core lab of BioClinica.

\section{Study scheme}

The trial profile of the PERFORM MRI project is shown in Fig. 1. Two MRIs were planned to be performed in each patient included in the study: the first at month 1 (M1),

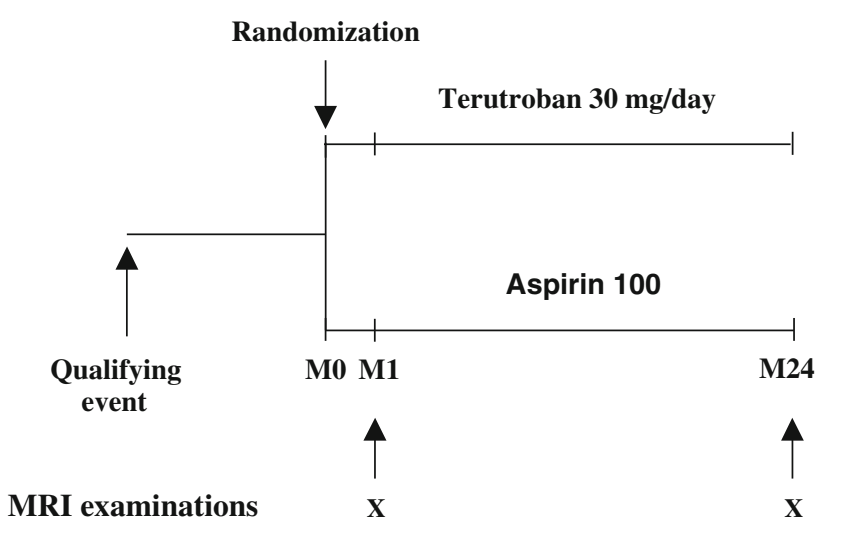

Fig. 1 The trial profile of the PERFORM MRI project

1-4 months after the qualifying clinical event and the second at month 24 (M24).

Participants were fully informed and written consent was obtained according to local regulatory requirements.

MRI acquisition

Before the initiation of the study, a total of 48 MRI sites from 16 countries (11 from Europe, and one each from Australia, Canada, Brazil, Singapore and Taiwan) were selected. MRI scans were performed on 1.5 Tesla scanners in 42 sites and on $3 \mathrm{~T}$ scanners in six sites.

The MRI protocol was implemented at each site following imaging parameter suggestions to ensure standardization across sites and different MRI systems, and to maximize consistency and comparability between data from different manufacturers. Numbers, vendors, models and field strengths of all MRI machines used are listed in Table 1. Each site was qualified using ACR phantom scans [8], to monitor image quality, in particular for geometrical distortions and adherence to the study protocol. The phantom scans were to be repeated yearly and after each significant software and hardware upgrade. Ten machines had a major hardware upgrade including system change in six cases, but due to phantom validation we could minimize as much as possible the effect of such changes that affected a relatively small number of patients. All scans included transverse fluid attenuated inversion recovery (FLAIR), gradient-echo T2*-weighted (T2*-weighted), diffusion weighted imaging (DWI) (three directions), 3D time-of-flight (TOF) MR angiography and high-resolution sagittal 3DT1 sequences. For sites with diffusion tensor imaging, transverse DTI (six directions) data were also performed. The total acquisition time was kept reasonably low (from 30 to $45 \mathrm{~min}$, according to the MRI system) to minimize patient's discomfort. Two-dimensional sequences were made of 5-mm thick contiguous slices with a $240 \mathrm{~mm}$ FOV and at least a $192 \times 256$ matrix $(128 \times 128$ 
Table 1 Numbers, vendors, models and field strengths of all MRI machines used

\begin{tabular}{llll}
\hline Number & Vendor & Model & Field \\
\hline 17 & GE & Signa Excite & 1.5 \\
2 & GE & Signa Excite & 3 \\
7 & GE & Signa HDx & 1.5 \\
5 & GE & Signa HDxt & 1.5 \\
5 & GE & Signa Horizon & 1.5 \\
1 & GE & Signa Infinity & 1.5 \\
5 & Philips & Achieva & 1.5 \\
1 & Philips & Achieva & 3 \\
9 & Philips & Intera & 1.5 \\
4 & Philips & Intera & 3 \\
1 & Philips & Intera Achieva & 1.5 \\
5 & Philips & Intera Achieva & 3 \\
1 & Philips & Gyroscan Intera & 1.5 \\
12 & Siemens & Avanto & 1.5 \\
6 & Siemens & Sonata & 1.5 \\
8 & Siemens & Symphony & 1.5 \\
7 & Siemens & Symphony Tim & 1.5 \\
1 & Siemens & Symphony Vision & 1.5 \\
3 & Siemens & Trio Tim & 3 \\
1 & Siemens & Vision Plus & 1.5 \\
1 & Picker/Marconi & Edge Eclipse & 1.5 \\
\hline
\end{tabular}

for DWI and DTI). TOF-MRA sequence was made of 1.6-mm thick slices with a $200 \mathrm{~mm}$ FOV and at least a $256 \times 256$ matrix. The 3DT1 protocol was chosen to follow ADNI recommendations [9] (1.2-mm thick slices, $240 \mathrm{~mm}$ FOV, $192 \times 192$ matrix), with slight modifications to reduce scan time.

Image management and reading sessions

All scans were centralized by BioClinica and quality was controlled to check for protocol compliance and image quality. In case of significant quality issues, repeat scans were requested. A MRI examination of insufficient quality at M1 excluded the patient from the study. For follow-up timepoints, only image data of sufficient quality were planned to be used for analysis.

After centralization and quality control and before submission to the readers, the image data were processed by the core lab. The aim of the image processing was to prepare the image data for centralized evaluation and to pre-detect the different structures of interest (such as white matter lesions, brain parenchyma, hippocampus) to improve the efficiency and the quality of centralized evaluations. This included various post-processing algorithms, e.g., 3D reformation, image registration, tissue segmentation, automated lesion detection to ensure best possible comparability. After these preparatory steps, image evaluations were carried out under supervision of BioClinica at remote sites on dedicated workstations by independent neuro-radiologists unaware of clinical information using a standardized procedure.

\section{MRI assessment}

Focal tissue changes related to the vascular pathology were to be assessed from M1 and M24 on FLAIR (new incidental infarcts and other white matter changes and changes of lesion volume), $\mathrm{T} 2 *$ (microbleeds identified as hypointense lesions) and DWI (incidental acute infarcts at M24). Changes in brain volume occurring between M1 and M24 to assess global brain atrophy and hippocampal were planned to be evaluated on the 3DT1 data set. Brain microstructural tissue changes were to be assessed from M1 and M24 on diffusion tensor imaging (DTI) using apparent diffusion coefficient (ADC) and fractional anisotropy (FA) maps.

\section{Lesion load}

Lesion load assessment on FLAIR sequences has been selected as FLAIR was previously recognized as more sensitive and specific than conventional or fast spin echo T2-weighted magnetic resonance imaging (MRI) for detecting lesions in the brain of patients with ischemic brain disease [10]. Its major advantages over $\mathrm{T} 2$ are a better sensitivity for small infarctions close to the sulci and a better differentiation between Virchow-Robin spaces, old lacunes and other white matter lesions [11]. Diffusion weighted imaging (DWI) was chosen as known to be very sensitive to detect acute ischemic stroke events [12].

Hyperintense FLAIR lesions, hypointense FLAIR lesions and hyperintense DWI lesions were pre-detected using an unsupervised 3D segmentation algorithm on FLAIR and DWI isotropic images after skull removal, and manually validated prior to submission to the readers (Fig. 2).

\section{Brain and hippocampal atrophy}

Brain parenchyma was pre-detected using a 3D segmentation algorithm on 3DT1 images after removal of the skull by $3 \mathrm{D}$ deformable registration of a brain atlas, and manually validated prior to submission to the readers (Fig. 3). Left and right brain volumes were derived from the total brain volume results using the interhemispheric plane as detected at M1 on sagittal 3DT1 images. These surrogate measurements allowed us to measure the impact of brain 
Fig. 2 Lesion detection. From left to right, hyperintense FLAIR lesions, hypointense FLAIR lesions, and hyperintense DWI lesions were pre-detected using an unsupervised 3D segmentation algorithm after skull removal, and manually validated prior to submission to readers
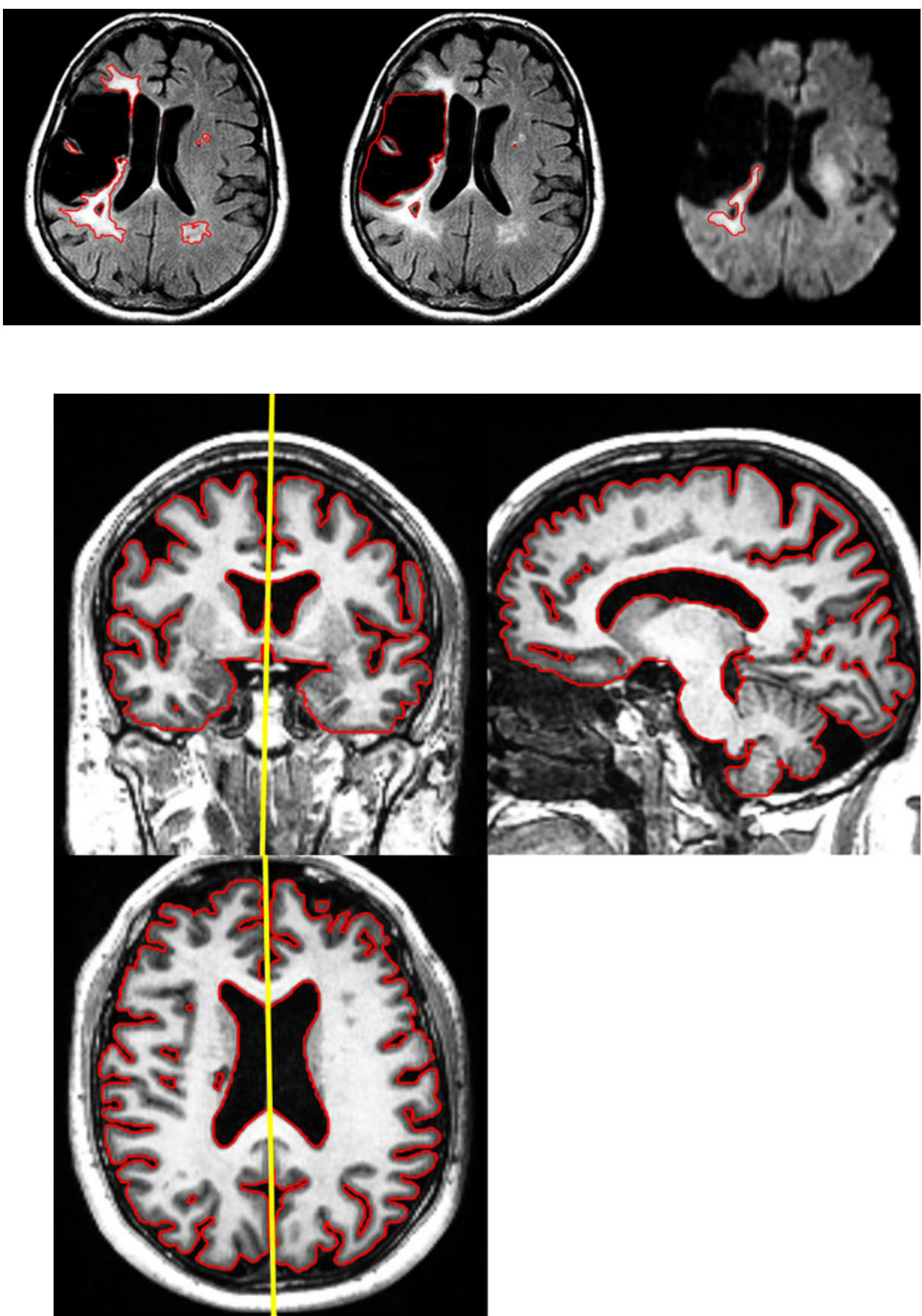

Fig. 3 Brain parenchyma detection. Brain parenchyma contours (in red) was predetected using a 3D segmentation algorithm on 3DT1 images by 3D deformable registration of a brain atlas, after skull removal, and manually validated prior to submission to the readers. Interhemispheric plane is shown in yellow atrophy with more accuracy on the contra-lateral hemisphere, as the initial infarct and surrounding tissue changes did not affect this measurement.

The Jacobian integration, a quantitative technique based on a 3D non-rigid matching of baseline and follow-up MRI examinations [13] was used for the assessment of the longitudinal brain volume changes. As a registration-based technique, it requires less manual interactions with respect to a segmentation-based technique or the boundary shift integral [14, 15] and allows the detection of small brain volume changes [16].

The hippocampal volume was defined as the complex interfolded layers of the cornu ammonis (grey matter), the dentate gyrus (grey matter), the alveus (white matter), the subiculum (grey matter) and the fimbria (white matter). The hippocampal-amygdalar boundary was identified with reference to the temporal horn of the ventricles and, where possible, to the presence of the alveus. Hippocampal 
Fig. 4 Hippocampus detection. Hippocampal contours (in red) were pre-detected using a semiautomatic technique prior to submission to the readers for confirmation

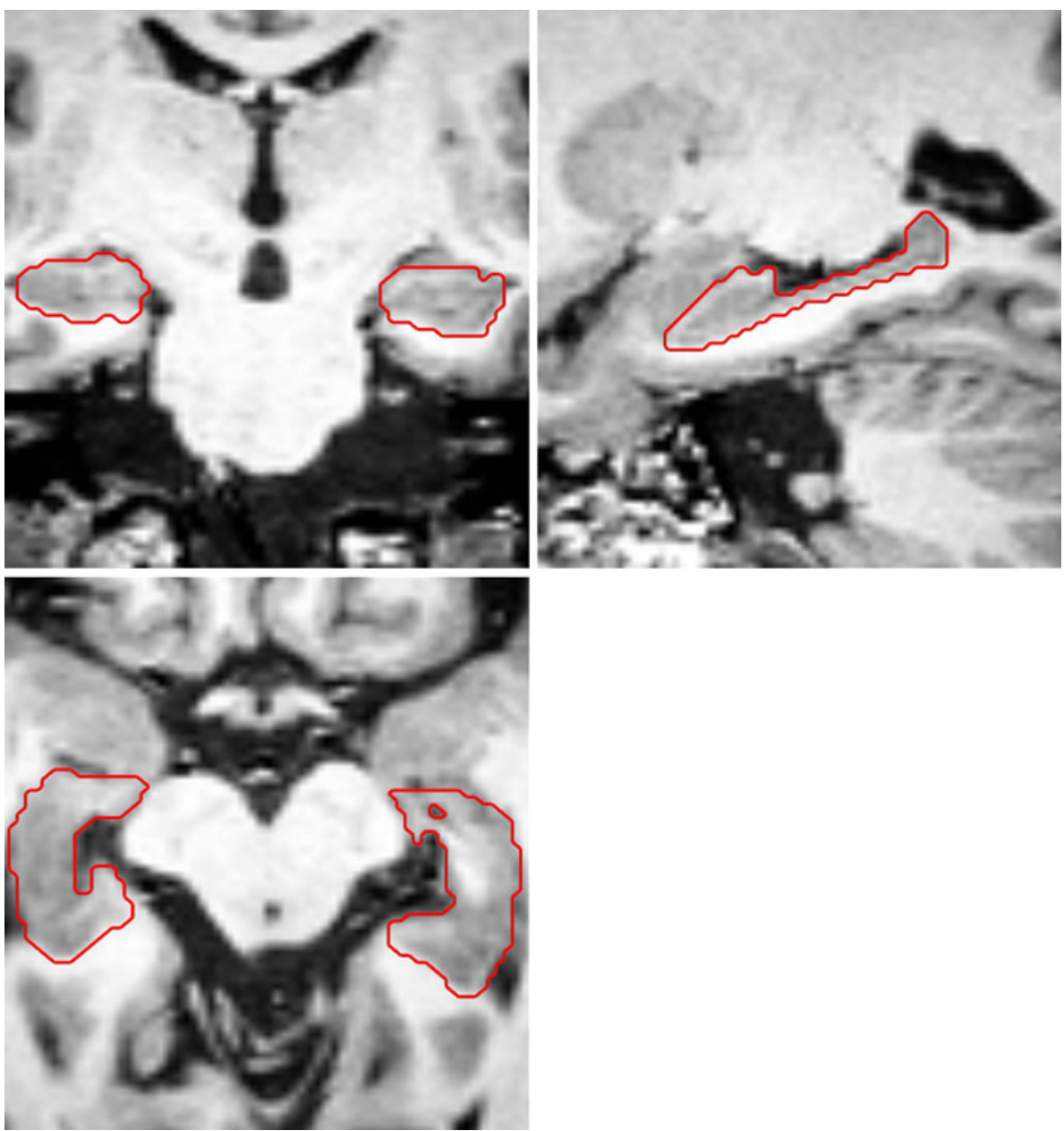

contours were pre-detected using a semi-automatic technique prior to submission to the readers for confirmation (Fig. 4).

For both brain parenchyma and hippocampal masks, 3DT1 data were displayed in a multiplanar reformatted mode so that the readers were able to use the sagittal (native), coronal and transverse orientations when correcting the pre-detected masks.

The volume of the intracranial cavity was used to normalize the different volumetric measurements. The $\mathrm{T} 2 *$ sequence was used for this purpose as it clearly depicts external CSF. Given that the full brain coverage could not be achieved for all patients (24-28 slices of $5 \mathrm{~mm}$ were used), the 3DT1 sequence was automatically coregistered to $2^{*}$ and used to manually complete for potentially missing upper and lower slices, and correct for $\mathrm{T} 2 *$-related artifacts (Fig. 5).

\section{Microbleeds}

Cerebral microbleeds $[17,18]$ were defined as punctuate, homogeneous, rounded, hypointense lesions smaller than
$5 \mathrm{~mm}$ in size, visualised on $\mathrm{T} 2 *$ sequences. They were counted throughout the brain by the readers (Fig. 6). Hypointense lesions in the subarachnoid space were considered likely to represent adjacent pial blood vessels and, therefore, were not included in the analysis. Symmetrical hypointensities in the globi pallidi (likely to represent calcifications or iron deposition) and flow voids from cortical vessels were also disregarded.

Cerebral microbleeds were also categorized using the following classification: cortical or cortico-subcortical and juxtacortical $(<5 \mathrm{~mm}$ from cortex) on one hand, or deep and infratentorial (including brainstem, cerebellum, thalamus, etc.) on the other hand.

\section{Brain microstructural tissue changes}

Diffusion tensor imaging (DTI) is a magnetic resonance imaging technique that measures the directionally dependent rate of water self-diffusion in each image voxel.

Subcortical white matter ischemic injury causes reductions in the oriented organization of white matter pathways 
Fig. 5 Intracranial cavity detection. The $\mathrm{T} 2 *$ sequence was used to detect the intracranial cavity contours (in $r e d)$ as it clearly depicts external CSF (left). Given the lack of full brain coverage for all patients the 3DT1 sequence was automatically coregistered to $\mathrm{T} 2 *$, resliced in the axial plane, and used to manually complete for potentially missing upper and lower slices (right)
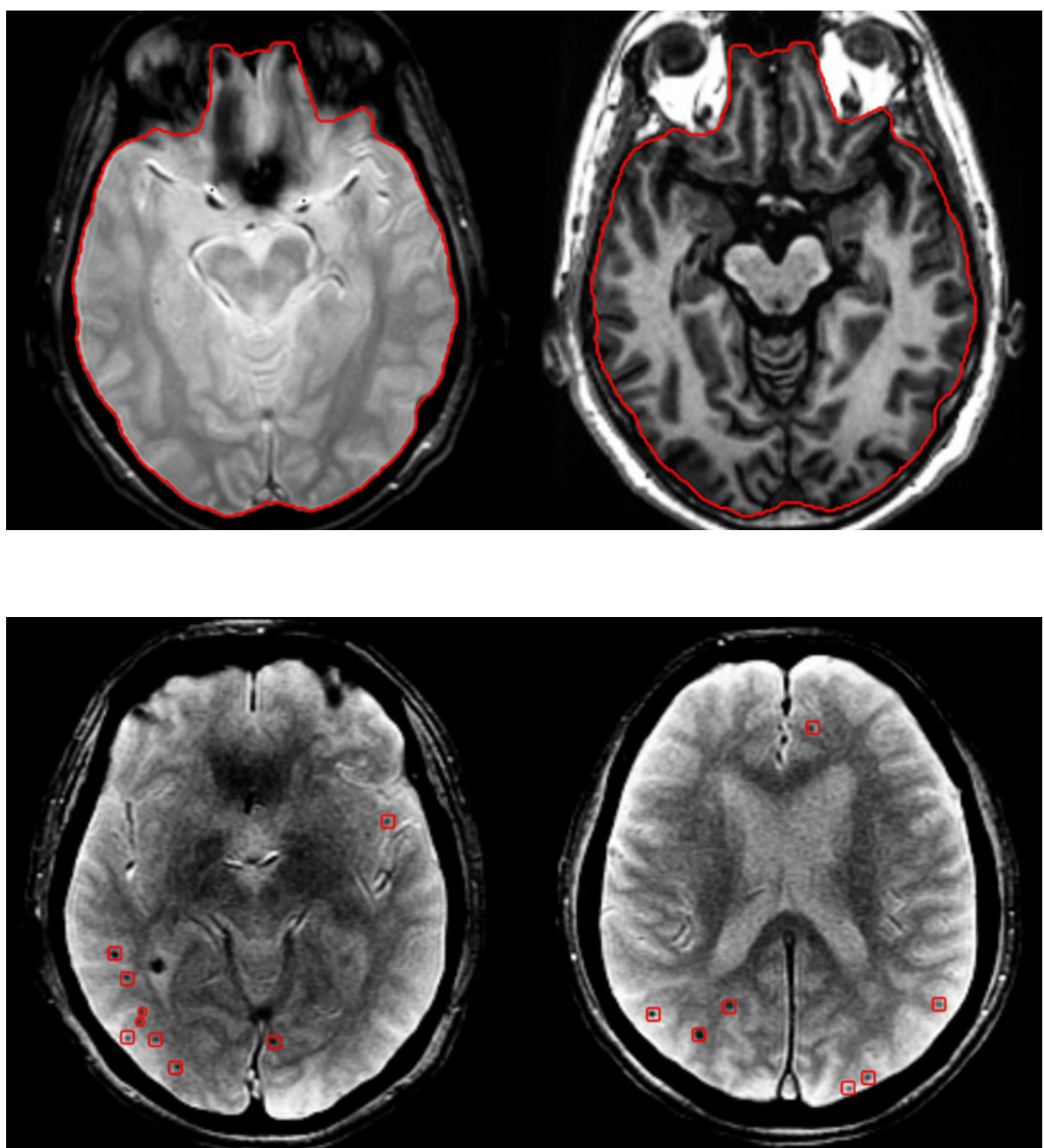

Fig. 6 Microbleeds assessment. Cerebral microbleeds, defined as punctuate, homogeneous, rounded, hypointense lesions smaller than $5 \mathrm{~mm}$ in size, visualised on $\mathrm{T} 2 *$ sequences, (in red), were counted throughout the brain by the readers, and classified as cortical, corticosubcortical, juxtacortical or deep and infratentorial

\section{Data management}

\section{Evaluation criteria}

The primary efficacy criteria of the study were lesion changes and brain volume changes between M1 and M24. All these parameters were assessed globally but also with regard to the side of the lesion of the initial ischemic event. The identification of the ipsilateral and contralateral hemispheres allows us to evaluate, in addition to the general progression of the cerebrovascular disease, the specific evolution of the disease in relation to the initial event.

As a criterion of interest for safety the number of emergent microbleeds was identified.

\section{Statistical analysis}

The PERFORM MRI project analyses will be under the supervision of the Biometry Division of the Institut de Recherches Internationales Servier. The analyses will be performed in all patients having taken the study drug and 
having M1 and M24 MRI examinations. Efficacy criteria will be analyzed in raw data as well as normalized data (normalization by the intracranial cavity volume) and will be expressed in change or relative change. Covariance analysis models adjusted for M1 value will be used to estimate the treatment effect, its 95\% CI and associated $p$ value.

The number of emergent microbleeds will be counted by treatment group and a $95 \% \mathrm{CI}$ of the treatment effect will be provided.

Inter- and intra-reader variability

Although all readers were highly qualified clinical stroke MRI readers, multiple readers were assigned to the same read tasks to ensure general consistency. In order to ensure the quality of evaluation results throughout the study and among the readers, the inter- and intra-reader reproducibility was monitored based on double reads of $5 \%$ of all evaluations. The criteria for acceptable intraand inter-variability rates were related to each type of MRI reading task. A threshold was chosen initially for each task and when the differences between two readers were higher than this threshold the readers were asked to repeat their reads after taking consensual decisions in order to improve reproducibility. When the variability was larger than expected, the concerned case was systematically reviewed and a consensual decision was made.

The following criteria for acceptable intra- and interrater variability rates between two double-readings were related to the MRI parameters. For global brain volumes a relative threshold of $1 \%$ of variability was accepted. For hippocampal volumes or for intracranial cavity volume, $5 \%$ of variability was accepted. For lesion volumes both a relative and an absolute thresholds were used: $5 \%$ of variability or an absolute volume variation of less than $300 \mathrm{~mm}^{3}$ was chosen for hyperintense FLAIR lesions, 3\% or $100 \mathrm{~mm}^{3}$ for hypointense FLAIR lesions, and $3 \%$ or $300 \mathrm{~mm}^{3}$ for hyperintense DWI lesions. Regarding microbleeds, these were categorized in four classes ( 0 bleeds, 12 bleeds, 3-10 bleeds, $>10$ bleeds) and two doublereadings were found as discrepant if their results led to classification in a different class.

\section{Population baseline characteristics}

Recruitment into the PERFORM MRI project started on February 22, 2006 and the last patient was randomized on February 25, 2008. A total of 1,056 patients were randomized from 48 MRI centers in 16 countries; $53 \%$ of the population was recruited in western Europe, $13 \%$ in
Table 2 Baseline characteristics of the PERFORM MRI study population

\begin{tabular}{ll}
\hline Total patients & 1,056 \\
Male, \% & 65 \\
Mean age, years & 68 \\
Age, \% & \\
$<65$ years & 39 \\
$\geq 65$ to <75 years & 39 \\
$\geq 75$ years & 22 \\
Ethnicity, \% & \\
Caucasian & 81 \\
Asian & 13 \\
Black & 4 \\
Other & 2 \\
Medical history, \% & \\
Hypertension & 84 \\
Hypercholesterolemia & 50 \\
Type II diabetes mellitus & 30 \\
Prior history of cerebral infarction & 11 \\
Angina pectoris & 6 \\
Hypertriglyceridemia & 6 \\
Prior history of TIA & 76 \\
Myocardial infarction & 7 \\
Peripheral artery disease & 7 \\
Previous treatments ${ }^{\mathrm{a}} \%$ & 3 \\
Statins & \\
Angiotensin-converting enzyme inhibitors & \\
& \\
Recorded between index strke and & \\
\hline
\end{tabular}

${ }^{a}$ Recorded between index stroke and randomization

Australia, $12 \%$ in Asia, $11 \%$ in Brazil, $8 \%$ in eastern Europe and $3 \%$ in Canada.

Demographic characteristics and past medical history are presented in Table 2. The mean age of the population was $67.7 \pm 8$ years (range 55-98 years), with $22 \%$ of patients over 75 years. Sixty-five percent of the population was male and $81 \%$ of Caucasian origin. The most frequent risk factors were hypertension (84\%), hypercholesterolemia $(50 \%)$, and diabetes $(30 \%)$. Eleven percent $(11 \%)$ of the population reported a previous ischemic stroke and $7 \%$ a previous TIA, while $7 \%$ had a history of myocardial infarction.

At baseline, after the qualifying stroke, $76 \%$ of the patients were receiving medical treatment with a statin, and $57 \%$ with an angiotensin-converting enzyme inhibitor.

Data relative to the qualifying event and disease characteristics are presented in Table 3.

Eighty-seven percent of the qualifying events were ischemic stroke. According to the pre-specified ischemic stroke classification, atherothrombotic or likely atherothrombotic stroke represents $69 \%$ of the ischemic stroke population and $26 \%$ had a lacunar stroke. 
Table 3 Qualifying event and stroke characteristics of the PERFORM study population

\begin{tabular}{ll}
\hline Qualifying event $(\%)$ & \\
Ischemic stroke & 87.4 \\
Transient ischemic attack & 12 \\
Arterial retinal ischemic event & 0.6 \\
Delay between qualifying event and randomization $(\%)$ & 30 \\
$\leq 1$ week & 40 \\
$>1$ week to $\leq 1$ month & 30 \\
$>1$ month & \\
Ischemic stroke subtypes $(\%)$ & 16 \\
Atherothrombotic stroke & \\
Likely atherothrombotic stroke & \\
Cardioembolic stroke & \\
Lacunar stroke & 53 \\
Coexisting & 1 \\
Unknown cause & 26 \\
Modified Rankin Scale $(\%)$ & 15 \\
Class 0 & 19 \\
Class 1 & \\
Class 2 & 28 \\
Class 3 & 36 \\
Class 4 & 23 \\
\hline
\end{tabular}

${ }^{a}$ Pure and coexisting cause

According to the Modified Rankin Scale scores at baseline, $87 \%$ of the patients had scores $\leq 2$, indicative of no or slight disability.

These data were comparable to the main study population characteristics [7].

\section{Conclusion}

The PERFORM MRI study is an exemplary study of a large-scale international multi-centre trial. Although such studies are expected, the complexity and difficulties at various levels have been hindering factors in the past. We describe the rationale, design and practical organization of a large stroke patient MRI trial. The project should provide important data on longitudinal brain disease progression with a follow-up of 2 years.

Conflict of interest The organization of the main PERFORM study has been described at length elsewhere [6]. The PERFORM MRI Project is supervised by the MRI Scientific Committee, comprising five members: Prof. M. Hennerici (Chairman), Prof. H. Chabriat, Prof. A. Gass, Prof. P. Maeder, and Dr. P. Michel. BioClinica, Lyon, France is responsible for the image quality control, the image processing and the reading of the MR images. The authors have all received honoraria, research grants, or both from Servier.
Open Access This article is distributed under the terms of the Creative Commons Attribution Noncommercial License which permits any noncommercial use, distribution, and reproduction in any medium, provided the original author(s) and source are credited.

\section{Appendix}

Principal investigators from MRI centres per country

Australia: C. Bladin, S. Davis, R. Helme, C. Levi, R. Schwartz; Austria: R. Schmidt, M. Vosko, J. Willeit; Belgium: P. Desfontaines, A. Michotte; Brazil: M. T. T. Da Silva, A. C. A. Ferraz, M. S. G. Rocha, J. R. C. B. Silveira; Canada: M. Hill, F. Silver, P. Teal; France: P. Clavelou, I. Crassard, T. De Broucker, C. Lamy, T. Moulin, C. Robin; Germany: J. Berrouschot, A. Grau, G. Hamann, L. Harms, R. Stingele, K. Szabo; Hungary: M. Szabo; Italy: C. Ferrarese, G. Neri, E. Parati; The Netherlands: W. M. Mulleners; Poland: J. Pniewski, K. Selmaj, A. Wajgt; Singapore: H. M . Chang, N. V . Ramani; Spain: A. Arboix Damunt, E. Diez Tejedor, O. Fernandez Fernandez, G. Izquierdo Ayuso, J. Roquer Gonzalez; Switzerland: P. Lyrer, P. Michel; Taiwan: K.-C. Chang; The United Kingdom: M. Dennis, K. Muir.

MRI project investigators by country

Australia: N. Ferris, S. Hudson, L. Masters, H. Prime, B. Tress; Austria: F. A. Fellner, S. Ropele, D. Zur Nedden; Belgium: T. Stadnik, L. Tshibanda; Brazil: L. Alberto Moreira De Souza, A. C. M. Maia Junior, M. S. Nacif, A. Sarmet Dos Santos; Canada: M. Heran, W. Hu, D. Mikulis; France: F. De Broucker, J. Gabrillargues, J-P. Guichard, Y. Narboux, C. Oppenheim, P. Wagner; Germany: A. Bormann, O. Jansen, R. Kern, R. Klingebiel, G. Layer, K. Wernecke; Hungary: E. Torok; Italy: E. Ciceri, M. Grimaldi, P. Ottaviano; The Netherlands: D. Venderink; Poland: M. Konopka, K. Selmaj, J. Walecki; Singapore: W. E. H. Lim, Y-Y. Sitoh; Spain: L. Ballenilla, C. Caparros-Escudero, A. Fernández Prieto, E. Mundt, J. Perich; Switzerland: P. Maeder, E-W. Radue; Taiwan: Y-L. Kuo; The United Kingdom: K. Muir, J. Wardlaw.

\section{References}

1. Neumann-Haefelin T, Moseley ME, Albers GW (2000) New magnetic resonance imaging methods for cerebrovascular disease: emerging clinical applications. Ann Neurol 47(5):559-570

2. Werring DJ, Frazer DW, Coward LJ et al (2004) Cognitive dysfunction in patients with cerebral microbleeds on $\mathrm{T} 2{ }^{*}$ weighted gradient-echo MRI. Brain 127:2265-2275 
3. Welch KM, Cao Y, Nagesh V (2000) Magnetic resonance assessment of acute and chronic stroke. Prog Cardiovasc Dis 43:113-134

4. Scheltens P, Barkhof F, Fazekas F (2003) White-matter changes on MRI as surrogate marker. Int Psychogeriatr 15(Suppl 1):261-265

5. Sorbera LA, Serradell N, Bolos J et al (2006) Terutroban sodium: prostanoid TP receptor antagonist, antithrombotic agent, antiatherosclerotic agent. Drugs Future 31:867-873

6. Bousser MG, Amarenco P, Chamorro A et al (2009) Rationale and design of a randomized, double-blind, parallel-group study of terutroban $30 \mathrm{mg} / \mathrm{day}$ versus aspirin $100 \mathrm{mg} /$ day in stroke patients: the Prevention of cerebrovascular and cardiovascular Events of ischemic origin with teRutroban in patients with a history oF ischemic strOke or tRansient ischeMic attack (PERFORM) study. Cerebrovasc Dis 27(5):509-518

7. Bousser MG, Amarenco P, Chamorro A et al (2009) The Prevention of cerebrovascular and cardiovascular Events of ischemic origin with teRutroban in patients with a history oF ischemic strOke or tRansient ischeMic attack (PERFORM) study: baseline characteristics of the population. Cerebrovasc Dis 27(6):608-613

8. Chen CC, Wan YL, Wai YY et al (2004) Quality assurance of clinical MRI scanners using ACR MRI phantom: preliminary results. J Digit Imaging 17(4):279-284

9. Jack CR Jr, Bernstein MA, Fox NC et al (2008) The Alzheimer's disease neuroimaging initiative (ADNI): MRI methods. J Magn Reson Imaging 27(4):685-691

10. Brant-Zawadzki M, Atkinson D, Detrick M et al (1996) Fluidattenuated inversion recovery (FLAIR) for assessment of cerebral infarction. Initial clinical experience in 50 patients. Stroke 27(7):1187-1191
11. Barkhof F, Scheltens $P$ (2002) Imaging of white matter lesions. Cerebrovasc Dis 13(2):21-30

12. Muir KW, Buchan A, von Kummer R et al (2006) Imaging of acute stroke. Lancet Neurol 5(9):755-768 Review

13. Vemuri BC, Ye J, Chen Y et al (2003) Image registration via level-set motion: applications to atlas-based segmentation. Med Image Anal 7(1):1-20

14. Freeborough PA, Fox NC (1997) The boundary shift integral: an accurate and robust measure of cerebral volume changes from registered repeat MRI. IEEE Trans Med Imaging 16(5):623-629

15. Boyes RG, Rueckert D, Aljabar P et al (2006) Cerebral atrophy measurements using Jacobian integration: comparison with the boundary shift integral. Neuroimage 32(1):159-169

16. Anderson VM, Fox NC, Miller DH (2006) Magnetic resonance imaging measures of brain atrophy in multiple sclerosis. J Magn Reson Imaging 23(5):605-618

17. Cordonnier C (2008) Brain microbleeds. Neurol 164(10):805808

18. Imaizumi T, Honma T, Horita $Y$ et al (2005) Dotlike hemosiderin spots are associated with past hemorrhagic strokes in patients with lacunar infarcts. J Neuroimaging 15(2):157-163

19. Jones DK, Lythgoe D, Horsfield MA et al (1999) Characterization of white matter damage in ischemic leukoaraiosis with diffusion tensor MRI. Stroke 30(2):393-397

20. Sullivan EV, Pfefferbaum A (2006) Diffusion tensor imaging and aging. Neurosci Biobehav Rev 30(6):749-761

21. Hagmann $P$, Jonasson L, Maeder $P$ et al (2006) Understanding diffusion MR imaging techniques: from scalar diffusion-weighted imaging to diffusion tensor imaging and beyond. Radiographics 26(1):S205-S223 